\title{
VARIAÇÃO NOS NÍVEIS DE HERBIVORIA FOLIAR EM DOIS MANGUEZAIS DA BAÍA DE PARANAGUÁ (PARANÁ - BRASIL)
}

\author{
IGNÁCIO, G.M. ${ }^{1 *}$; DOMINGUES, D. ${ }^{2}$; KOTLER, L. ${ }^{2}$; LANA, P.C. ${ }^{2} \&$ CARRILHO, J.C. ${ }^{2}$ \\ ${ }^{1}$ Fundação Universidade Federal do Rio Grande \\ ${ }^{2}$ Centro de Estudos do Mar - Universidade Federal do Paraná \\ "gabigmi@yahoo.com.br
}

\begin{abstract}
Ignácio, G.M.; Domingues, D.; Kotler, L.; Lana, P.C. \& Carrilho, J.C. 2005. Folivory variation in two mangroves of Paranaguá Bay (Paraná - Brasil). Braz. J. Aquat. Sci. Technol. 9(2):1-4. ISSN 1808-7035. Recent studies emphasize herbivory's importance in mangrove's carbon flux. Chemio- physical characteristics of the environment can influence leaf consumption, leading to differences in herbivory levels. This work evaluates possible differences in the degree of leaf consumption in two similarly structured mangrove forests submitted to different levels of pollution. In each forest, a $150 \mathrm{~m}$ transect was established perpendicularly to the waterline, and each one was divided in three $50 \mathrm{~m}$ strata. Thirty leaves from each of the local species (Rhizophora mangle, Avicennia schaueriana e Laguncularia racemosa) of mangrove were randomly collected, in a standardized way, in each strata. The samples were processed and herbivory levels were calculated through specific softwares. No differences were found between the forests nor between the stratum. The lack of differences between the stratum could be a consequence of the homogeneous distribution of the herbivorous in the forests. Furthermore, the fact that the forests present similar physiographic structures - in terms of tree's height, composition and distribution - can be ruling folivory levels, being more important than differences in soil and leaf composition.
\end{abstract}

Keywords: Folivory variation, mangroves, environmental impact, Paranaguá Bay.

\section{INTRODUÇÃO}

Estudos recentes têm enfatizado a importância da herbivoria no fluxo de carbono em manguezais, por acelerar os processos de formação de detritos e decomposição da matéria orgânica. A composição química das folhas pode ser influenciada pelas características químicas e físicas do ambiente, como grau de poluição ou nível de exposição/imersão (expresso pela distância da borda ou margem dos bosques), o que pode acarretar marcada variabilidade nos níveis de consumo de folhas de manguezal para manguezal. Lacerda et al (1986) encontraram diferenças significativas nas taxas de herbivoria em espécies diferentes de árvores. A variabilidade entre espécies também foi encontrada em outros estudos de herbivoria em manguezais (Odum et al., 1982). Vários estudos relatam diferenças nas composições químicas entre as espécies e suas possíveis causas ou conseqüências (Onuf et al., 1977; Coley, 1983; Lacerda et al., 1986; Buchsbaum \& Valiela, 1987; Duarte \& Cebrían, 1996; Godoy et al., 1997; Faraco \& Lana, 2004). O fósforo pode ser um importante determinante de tal variabilidade (Boto et al., 1984; Tomlinson, 1986 in Robertson, 1991; Feller, 1995). Outros estudos referem-se ao papel do conteúdo de nitrogênio das folhas no aumento da herbivoria, sugerindo que processos de eutrofização, pelo despejo de esgotos domésticos em áreas de manguezal, provavelmen- te afetam as taxas de herbivoria (Onuf et al., 1977; Vince et al., 1981 in Lacerda et al., 1986), idéia contestada por Johnstone (1981) e Lacerda et al (1986).

O objetivo deste trabalho foi avaliar diferenças nos níveis de herbivoria foliar em diferentes espécies de árvores e diferentes níveis de exposição de dois manguezais da Baía de Paranaguá, estruturalmente semelhantes, sendo um deles impactado e o outro nãoimpactado.

\section{Área de estudo}

Os manguezais estudados estão situados no setor polihalino do Complexo Estuarino da Baía de Paranaguá, no litoral do estado do Paraná, sul do Brasil. O clima local é subtropical, com verão chuvoso e inverno seco. As máximas pluviométricas ocorrem em fevereiro e as mínimas em julho e agosto. A precipitação anual média é de 2500 mm (máxima de 5300 mm), com a precipitação média durante a estação chuvosa sendo três vezes maior que na estação seca (Lana et al., 2000). As marés apresentam padrão semi-diurno, com amplitudes variando entre 0,5 e 2,0 metros (Lana $\&$ Guiss, 1991). As médias de salinidade variam entre 12-29 no verão (dezembro a março) e 20-34 no inverno (junho a agosto), enquanto a temperatura média da água varia entre $23-30^{\circ} \mathrm{C}$ e $18-25^{\circ} \mathrm{C}$, no verão e inverno, respectivamente (Lana et al., 2000). 
Os bosques de manguezais da região são geralmente compostos por apenas três espécies de árvores: Rhizophora mangle, Avicennia schaueriana e Laguncularia racemosa, com predominância de R. mangle (mais de $80 \%$ dos indivíduos), com altura média de 5 a 6 m. Os substratos são areno-síltico-argilosos.

O manguezal não-impactado situa-se na foz do rio Embocuí e o impactado entre a saída do canal do Anhaia e a empresa Fertilizantes Fosfatados S.A. (FOSPAR), na cidade de Paranaguá (Fig. 1). De acordo com Lana (2004), o manguezal impactado sofre: (1) desmatamento para expansão urbana, associado aos efeitos da poluição por esgotos ou por lixo sólido; (2) desmatamento para expansão industrial, portuária ou da rede de serviços (principalmente de energia elétrica), associado com os impactos de obras de infra-estrutura; (3) possibilidade de contaminação por petróleo, seus derivados e fertilizantes (principalmente fosfatados), devido à presença do porto e de terminais de combustíveis, além de contaminação por metais pesados e defensivos agrícolas. Informações sobre a qualidade ambiental da área de estudo estão disponíveis em CEM (1998).

\section{MATERIAL E MÉTODOS}

Em cada manguezal foi traçado um transecto de $150 \mathrm{~m}$ perpendicular à linha d'água, dividido em três estratos de $50 \mathrm{~m}$ cada. Em cada estrato foram aleato- riamente escolhidos três pontos. Em cada ponto escoIheu-se a árvore mais próxima de cada uma das três espécies, das quais foram coletadas aleatoriamente dez folhas de ramos terminais, entre 0,5 e 1,5 $\mathrm{m}$ de altura. As folhas amostradas foram digitalizadas em um scanner. A imagem binária resultante foi transformada em um arquivo vetorizado, formato .DXF, através do software ArcView. Cada folha vetorizada teve sua área total e área predada por herbivoria calculadas pelo software AutoCad. A partir dos valores encontrados calculou-se a percentagem de herbivoria foliar.

Como a amplitude dos valores percentuais foi muito grande (Figura 2), realizou-se uma transformação angular dos dados brutos (raiz quadrada do arco coseno), com o intuito de normalizar a distribuição e diminuir a variação nos desvios-padrão, pressupostos de uma análise de variância trifatorial que teve como objetivo avaliar a significância das diferenças nos níveis de herbivoria entre os dois manguezais, as três espécies de árvores e os estratos (ou distância da borda), considerados como fatores fixos. Constatadas diferenças significativas aplicou-se o teste post-hoc LSD.

\section{RESULTADOS E DISCUSSÃO}

Os níveis de herbivoria foliar não variaram significativamente entre manguezais ou estratos, mas a interação entre espécies de árvores e manguezais foi significativa (Figura 3). L. racemosa foi significativamente mais pastada do que $A$. schaueriana no manguezal

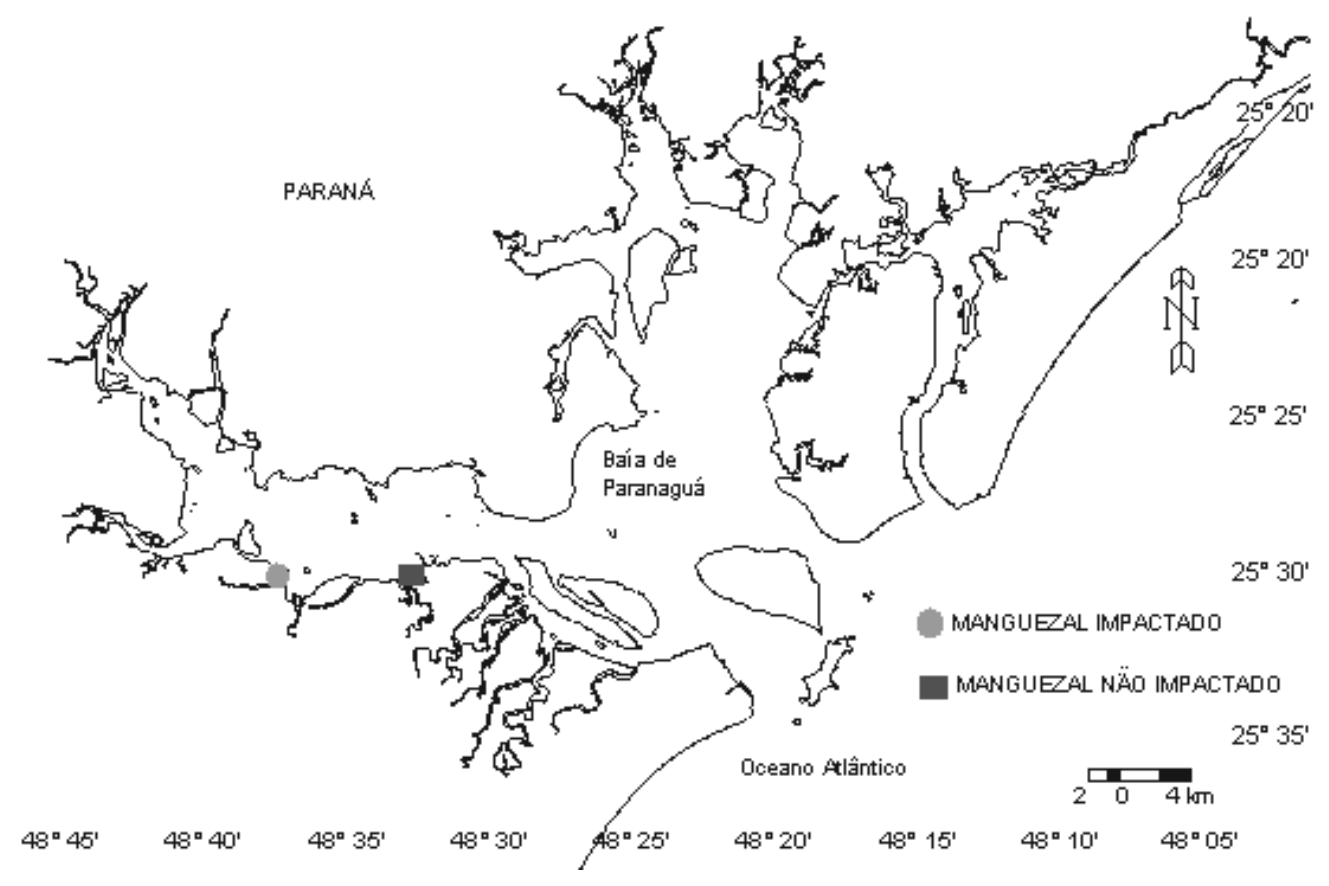

Figura 1 - Área de estudo. 


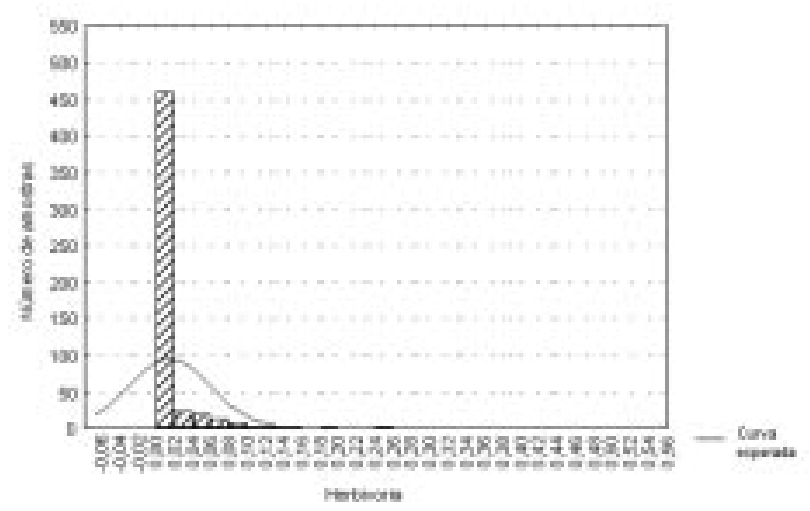

Figura 2 - Amplitude dos valores brutos de herbivoria foliar.

impactado. Por outro lado, R. mangle foi significativamente menos pastada do que $A$. schaueriana no manguezal não-impactado.

Os níveis de herbivoria aqui registrados são baixos quando comparados a estudos anteriores na Baía de Paranaguá (Brogim \& Lana, 1996; Faraco \& Lana, 2004), provavelmente pelo fato do estudo ter sido realizado em um período de clima atipicamente quente e seco (abril de 2002), o que inibe a atividade de herbívoros, como os caranguejos arborícolas.

A ausência de diferenças significativas nos níveis de herbivoria entre o manguezal impactado e o nãoimpactado não reflete necessariamente uma igualdade nas composições químicas e físicas do substrato e das folhas. Concluímos que as prováveis diferenças nas composições químicas dos bosques não estão influenciando a herbivoria. Segundo Lacerda et al (1986), uma relação direta entre o conteúdo de nitrogênio das foIhas e os níveis de poluentes nem sempre deve ser esperada. Nedwell (1975) demonstrou que o nitrogênio em excesso introduzido em manguezais por eutrofização é quase todo denitrificado, ficando indisponível para a absorção pelas folhas. Além disso, nem todo o nitrogênio das folhas está disponível para os herbívoros e sua qualidade (e.g., protéico, inorgânico) pode ser mais importante do que sua quantidade total (Mattson, 1988 in Lacerda et al., 1986). Outra possibilidade é que como os manguezais ocorrem em ambientes de alta salinidade, parte do nitrogênio estáve1 presente nas células como forma de controle osmótico, não contribuindo realmente para uma melhoria do status nutricional das folhas (Lacerda et al., 1986).

\section{CONCLUSÕES}

A ausência de diferenças nos níveis de herbivoria entre os estratos pode resultar da igualdade na distribuição populacional dos herbívoros predadores ao longo do manguezal. Além disso, o fato dos bosques apresentarem a mesma estrutura, em termos de altura, composição e distribuição das árvores, pode ser um condicionador primário dos níveis de herbivoria foliar,

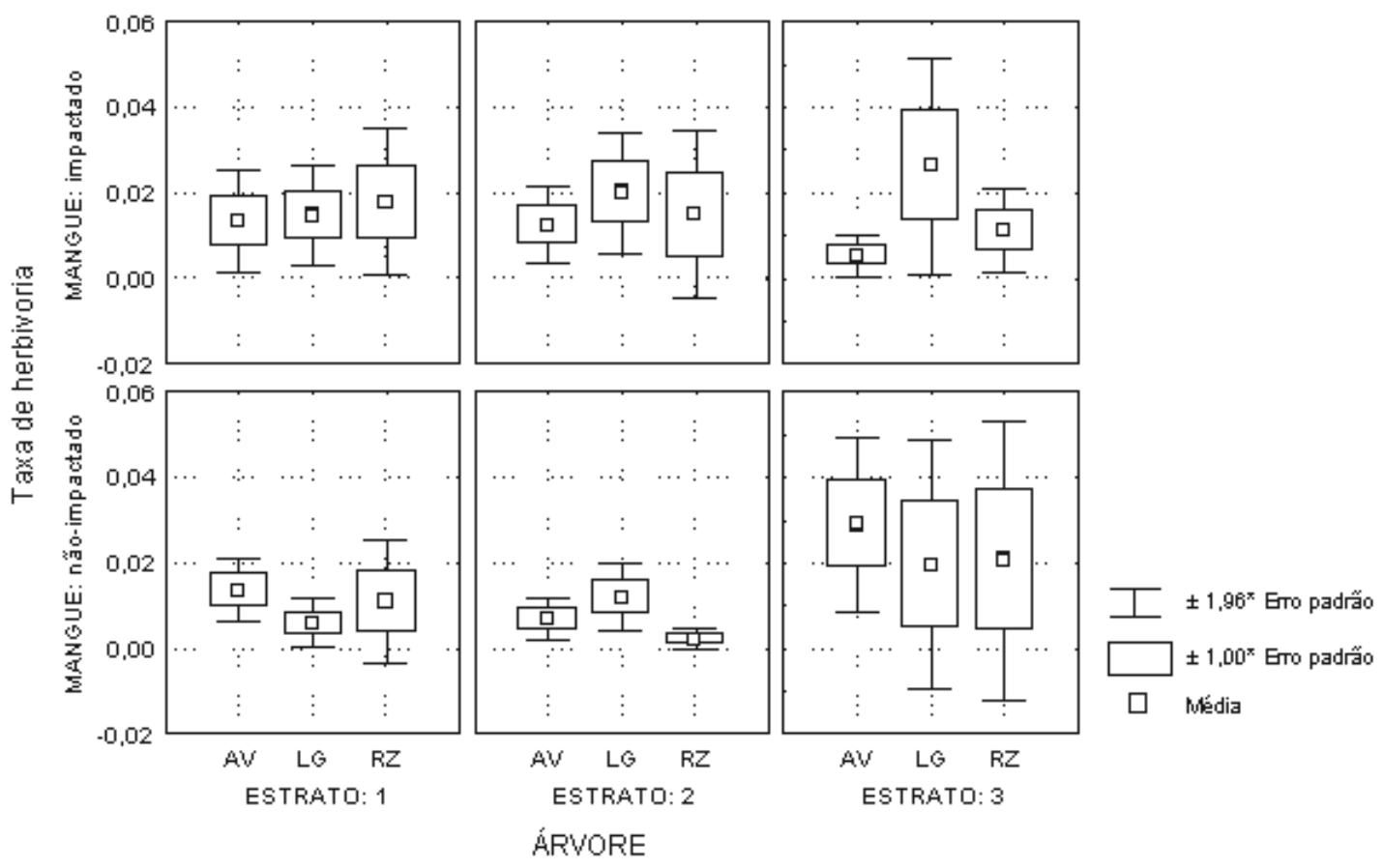

Figura 3 - Médias e desvios-padrão dos valores brutos de herbivoria. 
Ignácio et al.: Níveis de herbivoria foliar em manguezais da Baía de Paranaguá.

sobrepondo-se a eventuais diferenças nas características do substrato e das próprias folhas.

\section{REFERÊNCIAS}

Boto, K.G. \& Wellington, J.T. 1984. Soil characteristics and nutrient status in a northern Australian mangrove forest. Estuaries. 71: 61-69.

Brogim, R.A. \& Lana, P.C. 1996. Espectro alimentar de Aratus pisonii, Chasmagnathus granulata e Sesarma rectum (Decapoda - Grapsidae) em um manguezal da Baía de Paranaguá - Paraná. Iheringia. 83: 3543.

Buchbaum, R. \& Valiela, I. 1987. Variability in the chemistry of estuarine plants and its effect on feeding by Canada geese. Oecologia, Berlim. 73: 146-153.

CEM. 1998. Diagnóstico e prognóstico ambiental para construção do píer da FOSPAR - Fertilizantes Fosfatados S.A., Pontal do Sul.

Coley, P.D. 1983. Herbivory and defensive characteristics of tree species in a lowland tropical forest. Ecological Monographs. 53: 209-233.

Duarte, C.M. \& Cebrían, J. 1996. The fate of marine autotrophic production. Limnology and Oceanography. 41(8): 1758-1766.

Faraco, L.F.D. \& Lana, P.C. 2004. Leaf-comsuption levels in subtropical mangroves of Paranaguá Bay (SE Brazil). Wetlands Ecology and Management. 12: 115-122.

Feller, I.C. 1995. Effects of nutrient enrichment on growth and herbivory of dwarf red mangrove (Rhizophora mangle). Ecological Monographs. 65(4): 477-505.

Godoy, S.A.P.; Mayworm, M.A.S.; Kurt L.O.V.; Salatino, A. \& Shaeffer-Novelli, Y. 1997. Teores de ligninas, nitrogênio e taninos em folhas de espécies típicas do mangue. Revista Brasileira de Botânica. 20(1): 35-40.
Johnstone, I.M. 1981. Consumption of leaves by herbivores in mixed mangrove stands. Biotropica. 13: 252-259.

Lacerda, L.D.; José, D.V.; Rezende, C.E.; Francisco, M.C.F.; Wasserman, J.C. \& Martins, J.C. 1986. Leaf chemical characteristic affecting herbivory in a New World mangrove forest. Biotropica. 18(4): 350-355.

Lana, P.C. \& Guiss, C. 1991. Influence of Spartina alterniflora on structure and temporal variability of macrobenthic associations in a tidal flat of Paranaguá Bay (Southeastern Brazil). Marine Ecology - Progress Series. 73: 231-244.

Lana, P.C.; Marone, E.; Lopes, R.M. \& Machado, E.C. 2000. The subtropical estuarine complex of Paranaguá Bay, Brazil. p. 131-145. In: Seeliger, U. and Kjerfve, B. (eds.), Coastal Marine Ecosystems of Latin America, Ecological Studies. Vol. 144. Springer, Berlin, Germany.

Lana, P.C. 2004. Novas formas de gestão dos manguezais brasileiros, a Baía de Paranaguá como estudo de caso. Desenvolvimento e Meio Ambiente. 10: 169-174.

Nedwell, D.B. 1975. Inorganic nitrogen metabolism in a eutrophicated tropical mangrove estuary. Water Research. 9(2): 221-231.

Odum, W.E.; Mclvor, C.C. \& Smith III, T.J. 1982. The ecology of the mangroves of south Florida: a community profile. United States Fish and Wildlife Service, Office of Biological Services, Washington, D.C.

Onuf, C.P.; Teal, J.M. \& Valiela, I. 1977. Interactions of nutrients, plant growth and herbivory in a mangrove ecosystem. Ecology. 58(3): 514-526.

Robertson, A.I. 1991. Plant-animal interactions and the structure and function of mangrove forest ecosystems. Australian Journal of Ecology. 16: 433443. 